This item was submitted to Loughborough's Research Repository by the author.

Items in Figshare are protected by copyright, with all rights reserved, unless otherwise indicated.

\title{
Spin-orbital polarons in electron doped copper oxides
}

PLEASE CITE THE PUBLISHED VERSION

https://doi.org/10.1016/j.jmmm.2017.11.021

\section{PUBLISHER}

(c) Elsevier

\section{VERSION}

AM (Accepted Manuscript)

\section{PUBLISHER STATEMENT}

This work is made available according to the conditions of the Creative Commons Attribution-NonCommercialNoDerivatives 4.0 International (CC BY-NC-ND 4.0) licence. Full details of this licence are available at: https://creativecommons.org/licenses/by-nc-nd/4.0/

\section{LICENCE}

CC BY-NC-ND 4.0

\section{REPOSITORY RECORD}

Kusmartseva, Anna F., Heshan Yu, K. Jin, and F.V. Kusmartsev. 2019. "Spin-orbital Polarons in Electron Doped Copper Oxides". figshare. https://hdl.handle.net/2134/27691. 


\title{
Spin-orbital polarons in electron doped copper oxides
}

\author{
Anna Kusmartseva, ${ }^{1}$ Heshan $\mathrm{Yu}^{2}{ }^{2}$ Kui Jin, ${ }^{2,3}$ and F. V. Kusmartsev ${ }^{1}$ \\ ${ }^{1}$ Department of Physics, Loughborough University, \\ LE11 3TU Loughborough, United Kingdom \\ ${ }^{2}$ Beijing National Laboratory for Condensed Matter Physics, \\ Institute of Physics, Chinese Academy of Sciences, Beijing 100190, China \\ ${ }^{3}$ Collaborative Innovation Center of Quantum Matter, Beijing, 100190, China
}

\begin{abstract}
Present work demonstrates the formation of spin-orbital polarons in electron doped copper oxides, that arise due to doping-induced polarisation of the oxygen orbitals in the $\mathrm{CuO}_{2}$ planes. The concept of such polarons is fundamentally different from previous interpretations. The novel aspect of spin-orbit polarons is best described by electrons becoming self-trapped in one-dimensional channels created by polarisation of the oxygen orbitals. The one-dimensional channels form elongated filaments with two possible orientations, along the diagonals of the elementary $\mathrm{CuO}_{2}$ square plaquette. As the density of doped electrons increases multiple filaments are formed. These may condense into a single percollating filamentary phase. Alternatively, the filaments may cross perpendicularly to create an interconnected conducting quasi-one-dimensional web. At low electron doping the antiferromagnetic (AFM) state and the polaron web coexist. As the doping is increased the web of filaments modifies and transforms the AFM correlations leading to a series of quantum phase transitions - which affect the normal and superconducting state properties.
\end{abstract}


Multiple experimental evidences show that electron-doped cuprates are substantially different from their hole-doped counterparts ${ }^{1-6}$. Both systems are characterized by strongly correlated electrons and can be described by Hubbard-like models. The main differences arise with doping. Here, the main focus is given to lanthanum cuprates where electron and hole doping can be achieved by substituting $\mathrm{Ce}$ or $\mathrm{Sr}$ on the La sites of the parent compound $\mathrm{La}_{2} \mathrm{CuO}_{4}$ respectively. Electronically, $\mathrm{La}_{2} \mathrm{CuO}_{4}$ corresponds to an AFM state associated with a half-filled Hubbard band ${ }^{7,8}$. When the system is doped with holes, the resulting dominant electronic correlations are confined to the lower Hubbard band. On the other hand with electron doping the upper or second Hubbard band becomes more relevant. Furthermore, a mechanism is suggested to show how the addition of electrons may induce polarisation of the oxygen orbitals in the $\mathrm{CuO}_{2}$ plane.

In the parent compound $\mathrm{La}_{2} \mathrm{CuO}_{4}$ due to band half-filling the $\mathrm{AFM}$ order is attributed to half of the $\mathrm{Cu}$ sites having spin-up and the other half having spin-down orientations in the $\mathrm{CuO}_{2}$ plane. The main difference is that with hole doping some copper sites occupied by holes act effectively as Jahn-Teller ions ${ }^{9,10}$, which creates an interesting possibility for the formation of anti-ferroelectricity in hole-doped cuprates ${ }^{10,11}$. On the other hand, electron doping may result in completely filled $\mathrm{Cu}$ orbitals with electronic $d^{10}$ state corresponding to the upper Hubbard band. In contrast to the hole-doped case, the formation of the $d^{10}$ configuration is not associated with a Jahn-Teller lattice distortion.

Another key difference is that due to exchange forces the doped electron can only occupy copper sites with opposite spin orientation, which costs the large Hubbard energy $U \sim 10$ $\mathrm{eV}$. Here the situation with electron doping is similar to the Zhang-Rice singlet in holedoped cuprates $^{7}$. Specifically, due to the large on-site Hubbard repulsion $U$ the charge density cloud of the doped electron will shift away from the original $\mathrm{Cu}$ site, spreading out onto the neighbouring oxygen sites. This process will strongly polarise the oxygen orbitals, particularly those not directly involved in $p-d$ bonding. The orbital polarisation may extend to next-neighbouring oxygens resulting in the formation of a polarisation potential well spanning over several $\mathrm{Cu}$ sites with the same spin orientation (Fig.1a). The doped electron may become self-trapped by this polarisation well. Notably, the spin of the doped electron is different to the one located on the $\mathrm{Cu}$ sites inside the polarisation cloud.

To describe the formation of the spin-orbital polaron a schematic of the $\mathrm{CuO}_{2}$ plane is considered, where the $\mathrm{Cu}$ spin-up and spin-down sites are marked by black and green points 
(a)

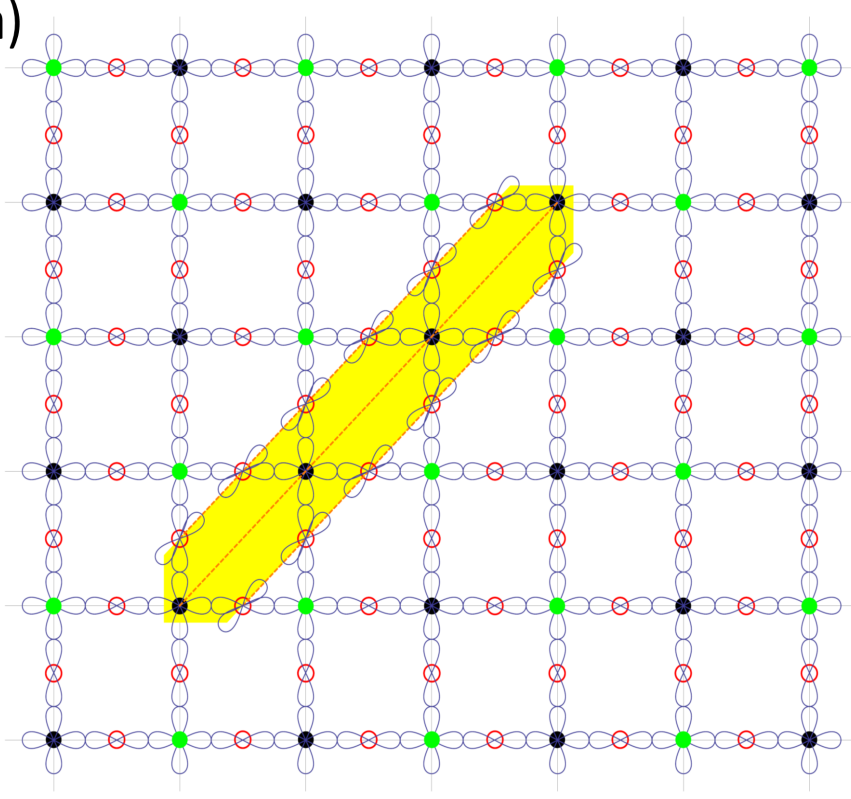

(b)

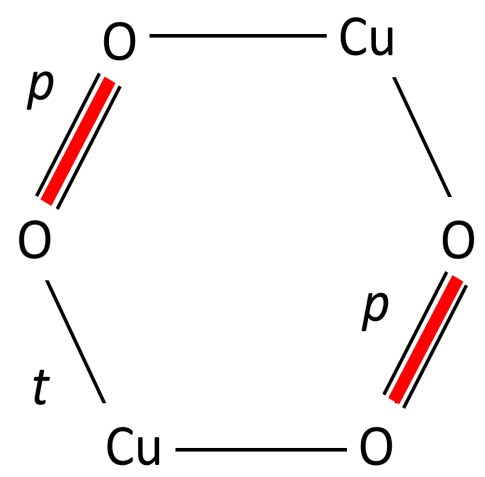

FIG. 1. a) A schematic representation of the spin-orbital polaron created in the $\mathrm{CuO}_{2}$ plane is shown. Its position is associated with the yellow polygon. The polaron arises due to the polarisation of the $p_{x}$ and $p_{y}$ orbitals on the oxygen atoms neighbouring the $\mathrm{Cu}$ sites by the doped electron. These polarised orbitals are denoted by the red dashed line. Here, for example, the polaron is localized across 4 distinct spin-up $\mathrm{Cu}$ sites (black points inside the yellow area). Usually, the polaron has elongated, quasi one-dimensional character and a needle-like shape. Within the polaron area the AFM order parameter $\Delta_{S D W}$ decreases. b) The lowest order and smallest size polaron occupies a hexagonal plaquette consisting of 4 oxygen and 2 copper atoms. A tight-binding model, involving the hybridisation and hopping integrals $p$ and $t$ respectively, is successfully used to describe the electronic correlations across these 6 sites.

respectively, while the oxygen atoms are represented by open red circles (see, Fig.1a). The electron cloud of the self-trapped doped electron extends from a single $\mathrm{Cu}$ site to the neighbouring oxygen atoms. The $p_{x}$ or $p_{y}$ oxygen orbitals which are perpendicular to the $p-d$ $\mathrm{Cu}-\mathrm{O}$ bonding become also polarised. Note, the polarisation is oriented along the diagonal in the $\mathrm{CuO}_{2}$ plane (Fig. 1a - red dashed line). In addition, the $p_{x}$ and $p_{y}$ orbitals may hybridise weakly to contribute to a non-zero, substantial overlapping integral, $p$ (Fig.1b). The combined orbital hybridisation and polarisation form a potential well for the doped 
electron, where it may become self-trapped over several $\mathrm{Cu}$ sites with the same spin orientation. Figure 1a shows a polaron (yellow coloured area) trapped over four spin-up Cu sites. The size of the smallest polaron involves two copper and four oxygen atoms spanning over a hexagonal plaquette (Fig.1b). It is important to note that problems related to orbital and spin-orbital polarons formation as well as the creation of polaron strings are rather topical and were actively discussed in the past, see e.g. refs $^{12-14}$. For example, the formation of spin-polarons in $\mathrm{La}_{2} \mathrm{CuO}_{4}$ was shown to originate with hole doping into the AFM ground state ${ }^{15,16}$. In transition metal oxides, specifically $\mathrm{LaMnO}_{3}$, orbital polarons were equally connected to hole doping of the orbital ground state. Here, all these ideas are combined to consider the creation of spin-orbital polarons arising due to oxygen orbital polarisation induced by electron doping, which is conceptually different from the case discussed in $\mathrm{LaMnO}_{3}$ and other systems ${ }^{12-17}$.

The formal description of the mechanism for the spin-orbital polaron and the conducting filament formation begins with the Heisenberg AFM state of the parent compound $\mathrm{La}_{2} \mathrm{CuO}_{4}$ which is well characterized by the Hubbard model at half-filling. To take into the account the polarisation of the oxygen orbitals the Zhang-Rice model ${ }^{7}$ is introduced:

$$
H=\sum_{i, \sigma} \epsilon_{d} C_{i \sigma}^{\dagger} C_{i \sigma}+\sum_{i, \sigma} \epsilon_{p} p_{i \sigma}^{\dagger} p_{i \sigma}+t \sum_{<i j>} C_{i \sigma}^{\dagger} p_{j \sigma}+h c+U \sum_{i} n_{i \uparrow} n_{i \downarrow}
$$

Here, the creation and annihilation operators of the doped electrons on the $\mathrm{Cu}$ and oxygen sites are denoted by $C_{i \sigma}^{\dagger}, C_{j \sigma}, p_{i \sigma}^{\dagger}$ and $p_{j \sigma}$ respectively. $t$ is the hopping integral between the $\mathrm{Cu}$ and $\mathrm{O}$ sites. The occupation number operators for spin up and spin down electrons are given by $n_{i \uparrow}=C_{i \uparrow}^{\dagger} C_{j \uparrow}$ and $n_{i \downarrow}=C_{i \downarrow}^{\dagger} C_{j \downarrow}$, respectively, while $U$ is the on-site Hubbard repulsion constant.

When describing the case of hole-doped cuprates Eq. 1 naturally transforms into the $t-J$ model, where the double occupancy of the copper sites is prohibited and the ZhangRice singlet is formed ${ }^{7}$. The situation is different when considering electron-doped cuprates. The most obvious distinction is that the second Hubbard band becomes now filled, which by definition implies doubly occupied $\mathrm{Cu}$ states at the cost of the Hubbard $U$. However, a doped electron can partially avoid the double occupancy by displacing its electron density to the neighbouring oxygens, resulting in two different effects. Firstly, this doping may induce a structural transition from the $T$ to the $T^{\prime}$ phase which has no apex oxygens. The 
second effect is related to the polarisation of free (non-bonded) oxygen orbitals triggered by the displacement of the charge density from the $\mathrm{Cu}$ site. Consequently if the charge deformation or the polarisation of the oxygen orbitals is taken into account, one may show that the hopping integral between the neighbouring oxygen sites increases. It is naturally assumed that the polarised orbitals on the neighbouring oxygens in the $\mathrm{CuO}_{2}$ plane lie along diagonals and become weakly hybridised. This leads to the appearance of a hopping path of the form $\mathrm{Cu}-\mathrm{O}-\mathrm{O}-\mathrm{Cu}$ where two next-neighbouring $\mathrm{Cu}$ sites are connected via two oxygen atoms (Fig. 1b). Such process provides substantial probability for the tunnelling of the doped electron between the next neighbouring $\mathrm{Cu}$ sites. In this case the original Hubbard model should be complemented by the term:

$$
H_{2}=\sum_{<<i j>>} t_{i j}^{\prime} p_{i \sigma}^{\dagger} p_{j \sigma}+h c
$$

where the value $t_{i j}^{\prime}$ is the hopping integral between the nearest oxygen sites arising only in the local presence of the doped electron and within the area where its electron density is confined. In general, if there is any hopping between the oxygen atoms in the plane its value is usually considered to be significantly smaller than $t$, that is $t_{i j}^{\prime}=t^{\prime} \sim 0.1 t$. The proposed model is conventionally named as $t-t^{\prime}$ Hubbard model and its phase diagram has been intensively discussed ${ }^{8}$. Here, the focus is shifted to the possible local enhancement of the $t^{\prime}$ term arising due to the local polarisation on the oxygen. In this case the hopping integral on the bonds, $<<i j>$, where the oxygen orbital polarisation occurs (e.g. $p_{x}$ and $p_{y}$ ), becomes equal to the value $t_{i j}^{\prime}=p$. Hence, the hopping term is substantially enhanced from its original, non-polarised value $p>>t^{\prime}$. In particular, it is proposed that the wave packet or charge density of the doped electron involved in polarising the oxygen orbitals definitely contributes to the value of $t^{\prime}$ hopping. The main effect is related to the formation of virtual hopping chains of the form $\mathrm{Cu}-\mathrm{O}-\mathrm{O}-\mathrm{Cu}$ which connect two $\mathrm{Cu}$ sites neighbouring along the diagonal of a $\mathrm{CuO}$ hexagonal plaquette. The polarised oxygen orbitals are key in this mechanism.

Importantly, the polarisation energy of the system is equal to $E_{p}=\alpha p^{2}$. Here $\alpha$ is a constant describing the polarisation rigidity of the system. It is playing the same role as elastic modulus in deformation energy of solids. Below we follow the logistics of the Pekar polaron theory (see, recent extensive review and the references therein ${ }^{18}$ ). We add the polarisation energy $E_{p}$ to the lowest eigenvalue $E(p)$ obtained from the solution of the 
Shrödinger equation $\left(H+H_{2}(p)\right) \Psi=E(p) \Psi$. Therewith we obtain the dependence of the polaron energy, $J_{\text {Pekar }}(p)=E(p)+E_{p}$, on the parameter $p$, describing the polarisation of the oxygen orbitals. In the simplest case of the smallest polaron the wave function, $\Psi$, describes an electron located on two copper and four oxygen sites, forming a hexagonal plaquette, see, the Fig.1b, superposed on an AFM background. The solutions of this equation gives the polaron energy, $J_{\text {Pekar }}(p)=E(p)+E_{p}$, which takes the form:

$$
J_{\text {Pekar }}(p)=\frac{1}{2}\left(-\sqrt{p^{2}+8 t^{2}}-p\right)+\alpha p^{2}
$$

here, for simplicity it is assumed that $\epsilon_{p}=\epsilon_{d}$ and $U=0$. The largest eigenvalue is most significantly affected by changes in these parameters. The function $J_{P e k a r}(p)$ has a clear minimum, the position of which $p_{\min }$ depends on the values of $t$ and $\alpha$. For example, when $\alpha=0.256 / t$ the value $p_{\min } \approx 1.41 t$. Although, this behaviour has an exact analytic expression, it is too expansive and non-compact to be included here. Instead, the approximation for $p_{\min }$ can be written as:

$$
p_{\min } \approx 1 / 4 \alpha+\sqrt{\left(1 /\left(1+32 t^{2} \alpha^{2}\right)\right)} / 4 \alpha,
$$

which becomes exact at large values of $32 t^{2} \alpha^{2}>1$. From Eq.3 it becomes obvious that the zero polarisation state, $p=0$, is unstable and its energy decreases when the parameter $p$ increases from zero to $p_{\min }$. Note, this is a universal phenomenon that is related to the polarisation of the oxygen orbitals. The value of the Hubbard $U$ associated with the onsite Coulomb repulsion at $\mathrm{Cu}$ sites has little to no effect on the position of the polarisation minimum $p_{\text {min }}$. This is because the electron density is mostly located around the four oxygen sites with little remaining around the Cu positions (Fig. 1a). In the ground state associated with the lowest eigenvalue the electron density shifts from the $\mathrm{Cu}$ sites towards oxygen sites when the value of the parameter $p$ increases. When $p>1$ the polaron charge density is mostly located on the oxygen sites. Therefore the on-site Coulomb Hubbard repulsion at $\mathrm{Cu}$ sites $U$ has almost no influence on its energy. Also the spin-orbital polaron is analogous to other types of spin polarons ${ }^{19-22}$. It has an opposite spin to the spins of the $\mathrm{Cu}$ sites and its orientation is elongated along the diagonal of the $\mathrm{CuO}_{2}$ square.

The formation of long conducting filaments is proposed to be linked to a condensation of such single spin-orbital polarons. Here many electrons become trapped in a single polarisation well, produced through the polarisation of the oxygen orbitals. The minimum of the 
(a)

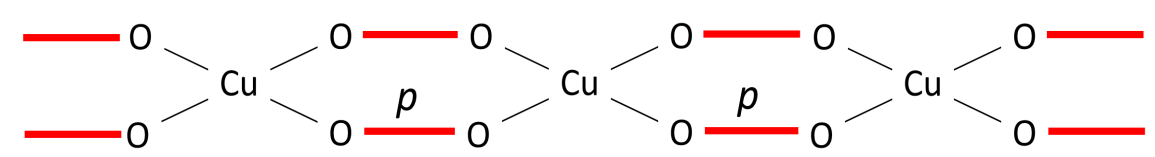

(b)

(c)
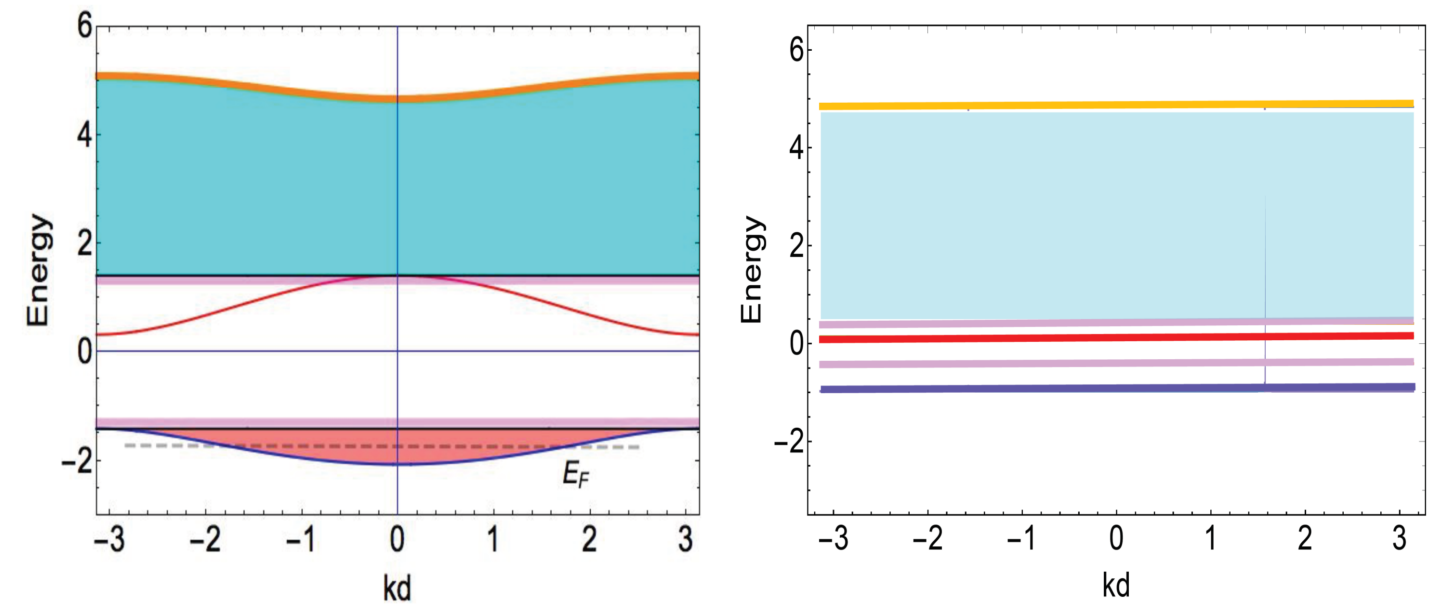

FIG. 2. a) A structure of the conducting filament. The unit cell has 5 atoms so there are 5 energy bands; b) an energy momentum relation of the conducting filament, for all five existing bands. It is calculated when the value of the polarisation is $p=-0.9 t$. Note that the lowest energy band has two minima at the boundary of the Brillouin zone (BZ). c) When one polarisation in the unit cell changes sign all 5 bands become completely flat. Such localisation is related to the formation of Aharonov-Bohm cage ${ }^{26,27}$.

Coulomb repulsion between electrons corresponds to a linear potential well, known as polaron string and stripe ${ }^{23-25}$. In this way, the electron motion is confined along a linear path across the $\mathrm{Cu}$ sites with the same spin orientation and connected by polarised oxygen sites. This type of transport is well described by a form of tight-binding model. An example of the structure of a conducting filament is shown in Fig. 2a. The electron spectrum obtained under those conditions is presented in Fig. 2b. The unit cell of the filament contains five atoms (one copper and four oxygens) consequently giving rise to five bands. Notably, there are two flat bands associated with localised polarons (when $E= \pm p$ ) and another three separate bands related to mobile polarons. The energy-momentum dependence for each of these bands, calculated with parameter value $p=0.9 t$, is given in the Fig.2b. The position of the lowest energy band is determined by the value of the hopping integral $t$. Its band- 
width depends on the value $p$ and the Hubbard parameter $U$. When the value $p$ increases from zero, the bandwidth increases. Conversely, when the value $U$ increases the bandwidth decreases. In fact, the combination of parameters $p$ and $U$ determines the size of the gap separating the first band from the second one. The gap vanishes when the value of $p$ reaches its critical value $p=\sqrt{2} t$. The energy of the bottom of the lowest band of the filament (its minimum is at $k=0$ ) decreases linearly with induced polarisation $p$. Recently, there have been many reported observations of anharmonicity ${ }^{28}$ or local charge-density wave (CDW) in electron-doped cuprates. ${ }^{29,30}$ These effects can be related to spin-orbital polarons or their ordered/condensed state as described here.

In summary, the new general concepts of conducting filaments and spin-orbital polarons have been introduced here within a theoretical framework. In the proposed scenario, the spin-orbital polarons form excitations of the conducting filamentary state. For the first time it was demonstrated that the conducting filaments and the spin-orbital polarons may coexist with an antiferromagnetic phase. The resultant electronic state is manifested as a filamentary spider web of one dimensional correlated electrons superposed on an antiferromagnetic background. The structure of conducting filaments is reminiscent of an electron liquid crystal or electron nematic ${ }^{31}$. The filaments form a microscopic electronic analogy to conventional liquid crystals. Moreover, the presence of spin-orbital polarons gives an additional degree of freedom to this picture. At high temperatures the filamentary web transforms into separate spin-polarons. In other words the electron liquid crystal melts into a viscous electronic, possibly, Luttinger liquid ${ }^{33,36}$. While at low temperatures and small electron doping the filaments and spin-orbital polarons can condense into a spider web that coexists with the AFM order. For electron-doped $\mathrm{La}_{2} \mathrm{CuO}_{4}$ this will occur at critical doping level $x<0.08$. The application of external magnetic field orders the filaments and makes the charge carriers more mobile, resulting in a combination of negative and positive magnetoresistance effects that are often observed in electron-doped cuprates ${ }^{5}$. The filamentary structure may also naturally describe the creation of a two-phase system important for the appearance of the magnetoresistance effects. ${ }^{34}$. Therefore, the electron-doped side of the phase diagram of cuprates can be consistently explained using these novel phenomenologies. The key of all these effects described here is polarisation of oxygen orbitals. The analogous important role of oxygen atoms have been also noticed for the vortex or magnetic flux trapping and paramagnetic Meissner effect observed in the hole-doped cuprates ${ }^{35-37}$. The similar 
frustrated electronic phase separation as described in this paper has been recently observed by using scanning micro x-ray diffraction and EXAFS methods in cuprates and iron based superconductors showing the emergence of a non Euclidean hyperbolic space for filamentary pathways at percolation ${ }^{30,38}$. The ideas proposed here may also offer new insights into the origin of superconductivity and other electronic instabilities reported in these systems.

Acknowledgements: The authors are very grateful to E.I.Rashba and B. Fine for illuminating discussions. The support and hospitality of Center for Theoretical Physics of Complex Systems, Daejeon, South Korea, where a part of this work has been done, is highly appreciated.

1 Ishii, K. et al. High-energy spin and charge excitations in electron-doped copper oxide superconductors. Nature Commun. 5, 3714 (2014).

2 Jin Kui, Zhang, X., Bach, P. and Greene, R. Evidence for antiferromagnetic order in $\mathrm{La}_{2-x} \mathrm{Ce}_{x} \mathrm{CuO}_{4}$ from angular magnetoresistance measurements. Phys. Rev. B 80, 012501 (2009).

3 Fournier, P. et al. Insulator-Metal Crossover near Optimal Doping in $\mathrm{Pr}_{2-2 x} \mathrm{Ce}_{x} \mathrm{CuO}$ : Anomalous Normal-State Low Temperature Resistivity. Phys. Rev. Lett. 81, 4720 (1998).

4 Dagan, Y., Qazilbash, M., Hill, C., Kulkarni, V. and Greene, R. Evidence for a Quantum Phase Transition in $\operatorname{Pr}_{2-x} \mathrm{Ce}_{x} \mathrm{CuO}_{4-\delta}$ from Transport Measurements. Phys. Rev. Lett. 92, 167001 (2004).

5 Yu, H., He, G., Jia, Y., Zhang, X., Yuan, J., Zhu, B., Kusmartseva, A., Kusmartsev, F.V. and Jin, K. Anomalous in-plane magnetoresistance of electron-doped cuprate $L a_{2-x} C e_{x} C u O_{4 \pm \delta}$. Science China Physics, Mechanics and Astronomy, 60, 097411 (2017).

6 Jin, K., He, G., Zhang, X., Maruyama, S., Yasui, S., Suchoski, R., Shin, J., Jiang, Y., Yu, H. S., Yuan, J., Shan, L., Kusmartsev, F. V., Greene, R. L. and Takeuchi, I., Anomalous magnetoresistance in the spinel superconductor LiTi2O4. Nature communications, 6, 7183 (2015).

7 F. C. Zhang, T. M. Rice, Phys. Rev. B37, 3760 (1988).

8 Hlubina, Richard. "Phase diagram of the weak-coupling two-dimensional t-t' Hubbard model at low and intermediate electron density." Physical Review B 59.149600 (1999).

9 G. Bednorz and A. Muller, Nobel lecture (Oct 1987); P. W. Anderson, The Theory of Superconductivity in the High-Tc Cuprate Superconductors (Princeton University Press, Princeton, 
1997).

10 F. V. Kusmartsev and M. Saarela, Dipolar clusters and ferroelectricity in high T-c superconductors, Int. J. Mod. Phys. B29 (SI),1542002 (2015); DOI: 10.1142/S0217979215420023

11 Saarela, M. and Kusmartsev, F.V., Phase transitions to dipolar clusters and charge density waves in high T c superconductors. Physica C: Superconductivity and its Applications, 533, pp.9-19 (2017).

12 T. Mizokawa, D.I. Khomskii, and G. A. Sawatzky, Phys. Rev. B 63, 024403 (2001).

13 K. Wohlfeld, A.M. Oles, and P. Horsch, Phys. Rev. B 79, 224433 (2009).

14 K. Wohlfeld, "Spin, orbital, and spin-orbital polarons in transition metal oxides", AIP Conference Proceedings 1162, 220 (2009).

15 G. Martinez and P. Horsch, Phys. Rev. B 44, 317 (1991).

16 J. van den Brink, P. Horsch, and A. M. Oles, Phys. Rev. Lett 85, 5174 (2000).

17 Saarela, M., and F. V. Kusmartsev. "Bound clusters and pseudogap transitions in layered highTc superconductors." J of Supercond. and Novel Magn. 28 : 1337-1341(2015).

18 Dykman, Mark I., and Emmanuel I. Rashba. "The roots of polaron theory." Physics Today 68.4, 10 (2015).

19 M.A. Krivoglaz, Sov. Phys.-Usp., 16, 856 (1974)

20 E. I. Nagaev, Physics of Magnetic Semiconductors (Mir Publishers, Moscow, 1983).

21 Schrieffer, J.R., Wen, X.G. and Zhang, S.C. Spin-bag mechanism of high-temperature superconductivity. Phys. Rev. Lett. 60, 944-947 (1988).

22 N. Mott and Z. Zinamon, Rep. Prog. Phys. 33, 81 (1970)

23 F.V. Kusmartsev, J. de Physique IV 9, Pr10-321 (1999)

24 F.V. Kusmartsev, Phys. Rev. Lett. 84, 530 (2000); Phys. Rev. Lett. 84, 5026 (2000).

25 F.V. Kusmartsev, D. Di Castro, G. Bianconi and A. Bianconi," Transformation of Strings into Stripes and itinerant Free Carriers in HTSC", Phys. Lett. A275, 118 (2000)

26 Vidal, J., Mosseri, R. and Doucot, B., Aharonov-Bohm cages in two-dimensional structures. Physical review letters, 81(26), 5888 (1998).

27 Kusmartsev, F. V. Fine structure and fractional M/N Aharonov-Bohm effect. Physical Review B, 52(20), 14445 (1995).

28 Menushenkov, A.P., Kuznetsov, A.V., Chernikov, R.V. et al. J Supercond Nov Magn 27, 925 (2014). 
29 Eduardo H. da Silva Neto, Sci Adv., 2: e1600782. (2016); doi: 10.1126/sciadv.1600782.

30 G. Campi, A. Bianconi, N. Poccia, et al., Nature 525, 359 (2015).

31 S. A. Kivelson, E. Fradkin, V. J. Emery, Electronic liquid-crystal phases of a doped Mott insulator. Nature 393, 550-553 (1998).

32 F.V.Kusmartsev, A. Luther and A. Nersesyan, To the theory of two- dimensional Luttinger liquid. JETP Lett. 55, 724 (1992).

33 A.A. Nersesyan, A. Luther and F.V.Kusmartsev, Scaling properties of the two-chain model, Physics Letters A176, 363 (1993).

34 Bulgadaev S, Kusmartsev F V, Large linear magnetoresistivity in strongly inhomogeneous planar and layered systems. Physics Letters A 342(1), 188-195 (2005).

35 Rykov, A.I., Tajima, S. and Kusmartsev, F.V., . High-field paramagnetic effect in large crystals of $\mathrm{YBa}_{2} \mathrm{Cu}_{3} \mathrm{O}_{7-\delta}$. Physical Review B, 55(13), 8557 (1997).

36 Kusmartsev, F. V., Orbital glass. Physics Letters A, 169(1-2), 108-114 (1992).

37 Rykov, A.I., Tajima, S., Kusmartsev, F.V., Forgan, E.M. and Simon, C. Change in the order of the melting transition with oxygen content in $\mathrm{YBa}_{2} \mathrm{Cu}_{3} \mathrm{O}_{7-\delta}$. Physical Review $\mathrm{B}, 60(10)$, 7601 (1999).

38 G. Campi, A. Bianconi. Journal of Superconductivity and Novel Magnetism 29, 627 (2016). 